\title{
Experimental study by full field measurement techniques of the stress gradients effect under fretting, fretting-fatigue and notch fatigue
}

\author{
By A-R. Moustafa*, B. Berthel*, E. Charkaluk**, S. Fouvry*
}

*LTDS, UMR 5513, Ecole Centrale de Lyon, 36 Avenue Guy de Collongue, Ecully 69134 Cedex, France, abdel-rahman.moustafa@ec-lyon.fr, bruno.berthel@ec-lyon.fr, siegfried.fouvry@ec-lyon.fr

**LML, UMR 8107, Ecole Centrale de Lille, boulevard Paul Langevin, 59655 Villeneuve d'Ascq Cedex, France, eric.charkaluk@univ-lille.fr

\section{Abstract}

In this work, we propose an experimental study of the stress gradients effect under Fretting, FrettingFatigue and notch fatigue loadings using innovative techniques such as infrared measurements and digital image correlation. A new non-destructive thermal method for the prediction of crack nucleation thresholds is developed and the predictions are compared with destructive results.

\section{Introduction}

One of the main causes of structure failure under cyclic loadings is the presence of high stress gradients states. As illustrated in figure 1, Fretting, a localized relative motion between components pressed together by external normal forces and undergoing oscillating tangential forces. Along with Fretting-Fatigue, a contact stressing and a quasi-homogeneous fatigue loading, can be considered as the worst case scenario which introduces the strongest stress gradients. Taking into account the effects of those gradients on the mechanical behavior is essential for a proper evaluation of structure's life. Considerable progress has been made in the understanding of this phenomenon but the classical identification techniques used [1] remain time-consuming and require expensive destructive methods that render dispersive results. Alternative identification methods based on the material's self heating response where developed to provide fatigue limits under unixial $[2,3,4,5]$ and multiaxial [6] loadings.

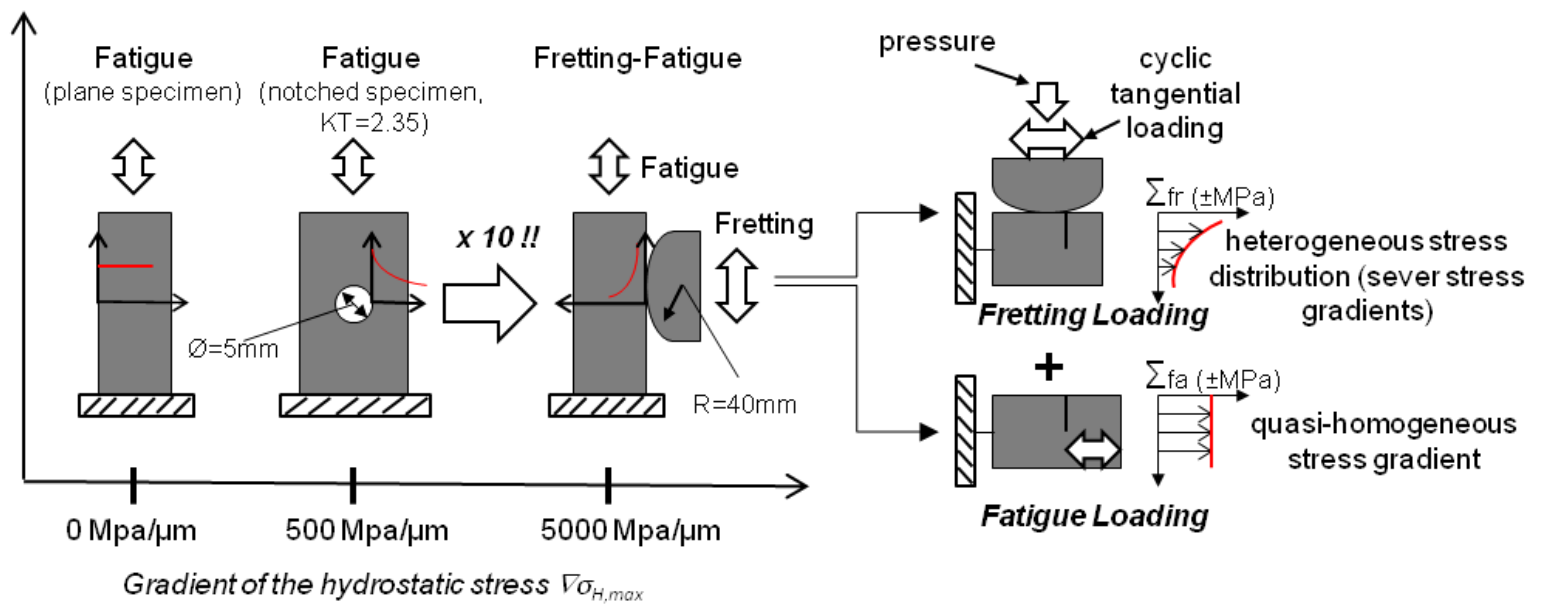

Fig. 1. Illustration of the stress gradient conditions [7]

This work emphasizes the application of these alternative methods using innovating techniques such as infrared measurements and digital image correlation to evaluate the crack nucleation threshold. Crack nucleation response at $10^{6}$ cycles of a well known $35 \mathrm{Ni} \mathrm{Cr}$ Mo 16 low-alloyed steel was studied under various plain fretting and fretting fatigue elastic partial slip conditions, another C70 steel alloy notched fatigue specimens with a centered hole were also considered, covering a wide stress gradient domain.

\section{Material and experiments}

\subsection{Materials}

The present study was performed on a $35 \mathrm{Ni} \mathrm{Cr}$ Mo 16 low-alloyed steel, in a cylinder on flat contact configuration. Cylindrical pads are made from a heat treated steel alloy $100 \mathrm{C} 6$ with a controlled roughness $(\mathrm{Ra}=$ $0.4 \mu \mathrm{m}$ ), ensuring that cracks arise only in plane and fatigue specimens. Table 1 shows the mechanical and fatigue properties of the specimens used in this work 


\begin{tabular}{|l|c|c|c|c|}
\hline Material & $\mathrm{E}(\mathrm{GPa})$ & $v$ & $\sigma_{\mathrm{u}}(\mathrm{MPa})$ & $\sigma_{\mathrm{y} 0.2}(\mathrm{MPa})$ \\
\hline 35NCD16 & 200 & 0.3 & 1130 & 810 \\
\hline $100 \mathrm{C6}$ & 195 & 0.3 & 1500 & 813 \\
\hline
\end{tabular}

Table 1. Mechanical and fatigue properties of the studied specimens.

\section{Experimental device and loading conditions}

Tests were carried out using experimental set-ups specially designed at LTDS and mounted on a servohydraulic machines. Figure 2 and table 2 illustrate the experimental strategy and conditions applied to quantify respectively the fretting and the fretting fatigue cracking process.

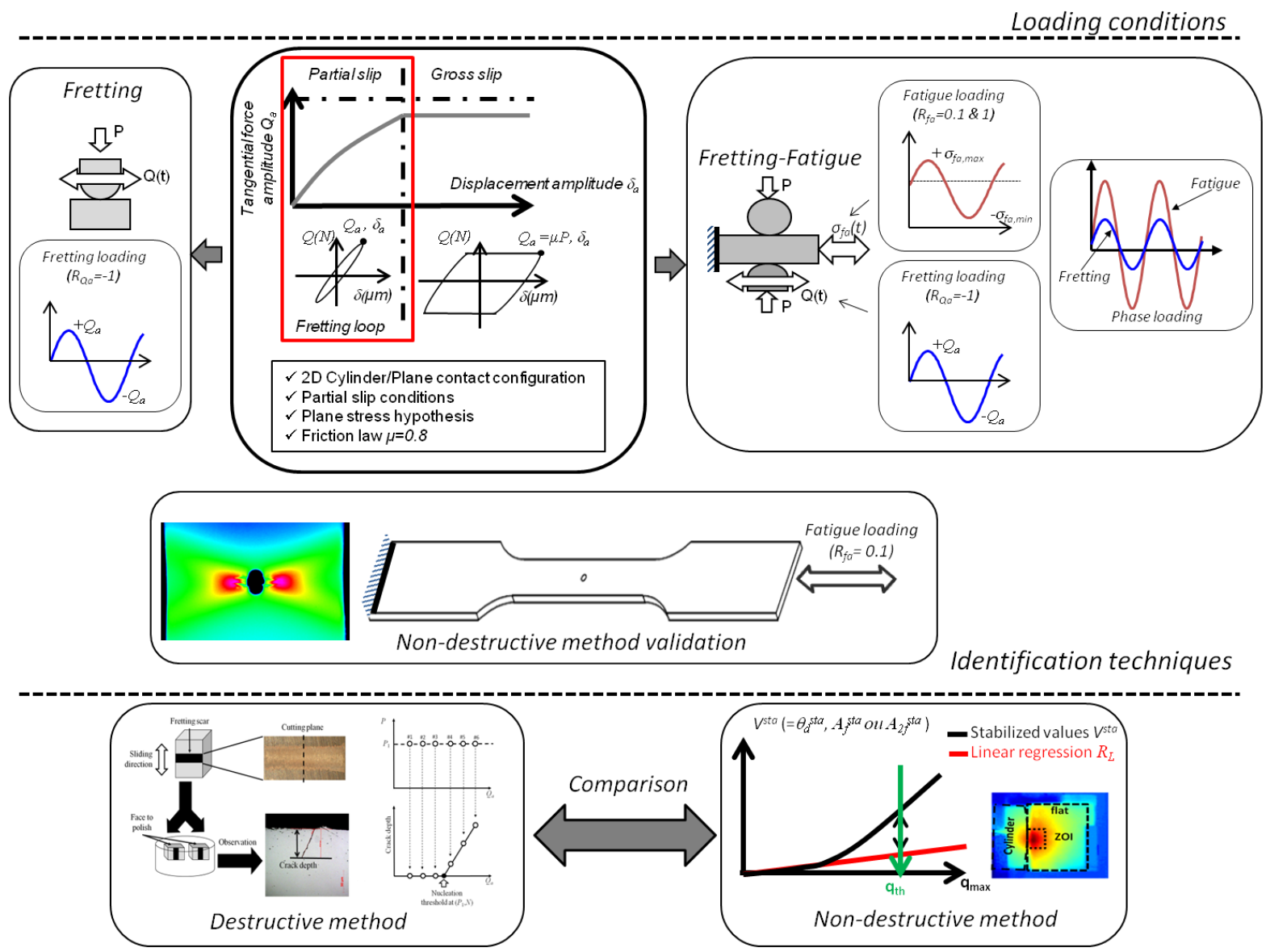

Fig. 2. Experimental strategy used to quantify the cracking process

\subsection{Fretting tests}

A fretting test consists of applying a static normal force $P$, followed by an cyclic displacement $\delta$, generating a cyclic tangential load $Q$ on the contact zone [8]. P, Q and $\delta$ are recorded during the tests and the $\delta-Q$ fretting loop are plotted and monitored to maintain a partial slip contact configuration. (figure 2)

\subsection{Fretting fatigue tests}

The Fretting-fatigue experiments were performed using a dual actuator device [9] as in [10, 11] allowing the application of the fretting and the fatigue loadings separately. This system allows the application of combined phase and unphased loadings. In this present work, only phase loadings were investigated. Fatigue loadings ( $\sigma_{f a, \max }$ maximum fatigue stressing, $\sigma_{f a, \min }$ minimum fatigue stressing and $R_{f a}=\frac{\sigma_{f a, m i n}}{\sigma_{f a, m a x}}$ ) along with contact loading $(\mathrm{P}, \mathrm{Q}, \delta)$ characteristics are recorded all along the tests. 


\begin{tabular}{|ccccccccc|}
\hline & $R(\mathrm{~mm})$ & $L(\mathrm{~mm})$ & $P_{\text {max }}(\mathrm{MPa})$ & $P(\mathrm{~N} / \mathrm{mm})$ & $\sigma_{\max , \mathrm{fa}}(\mathrm{MPA})$ & $R_{f a}$ & $\mu_{t}$ & $Q_{d}(\mathrm{~N} / \mathrm{mm})$ \\
\hline F_1 & 20 & 8 & 800 & 349 & 0 & 0 & 0,8 & 184 \\
F_2 & 20 & 8 & 1000 & 545 & 0 & 0 & 0,8 & 215 \\
F_3 & 40 & 8 & 600 & 392 & 0 & 0 & 0,85 & 271 \\
F_4 & 40 & 8 & 800 & 697 & 0 & 0 & 0,85 & 287 \\
F_5 & 40 & 8 & 1000 & 1089 & 0 & 0 & 0,85 & 310 \\
F_6 & 80 & 8 & 400 & 349 & 0 & 0 & 0,8 & 217 \\
F_7 & 80 & 8 & 600 & 784 & 0 & 0 & 0,8 & 305 \\
F_8 & 80 & 8 & 800 & 1394 & 0 & 0 & 0,8 & 399 \\
F_9 & 80 & 8 & 1000 & 2178 & 0 & 0 & 0,8 & 470 \\
F_10 & 160 & 12 & 400 & 697 & 0 & 0 & 0,8 & 320 \\
F_11 & 160 & 12 & 600 & 1568 & 0 & 0 & 0,8 & 510 \\
\hline FF_1 & 80 & 8 & 600 & 795 & 200 & 0.1 & - & 261 \\
FF_2 & 80 & 8 & 600 & 795 & 400 & 0,1 & - & 137 \\
FF_3 & 80 & 8 & 600 & 795 & 200 & 1 & - & 300 \\
FF_4 & 80 & 8 & 600 & 795 & 400 & 1 & - & 278 \\
\hline
\end{tabular}

Table 2. Loading conditions ( $F$, for Fretting and FF, for Fretting-fatigue)

\section{Classical crack nucleation identification technique: Destructive Methode}

This technique consist on testing several tangential load amplitudes, $Q_{a}$, for a given normal load $\mathrm{P}$ [1]. The sample is cut along the middle plane perpendicular to the fretting loading. The new surfaces are then polished and observed with an optical microscope to measure the crack length and depth (figure 3 ). This process is repeated at least three times in order to assess the homogeneity of the crack data.
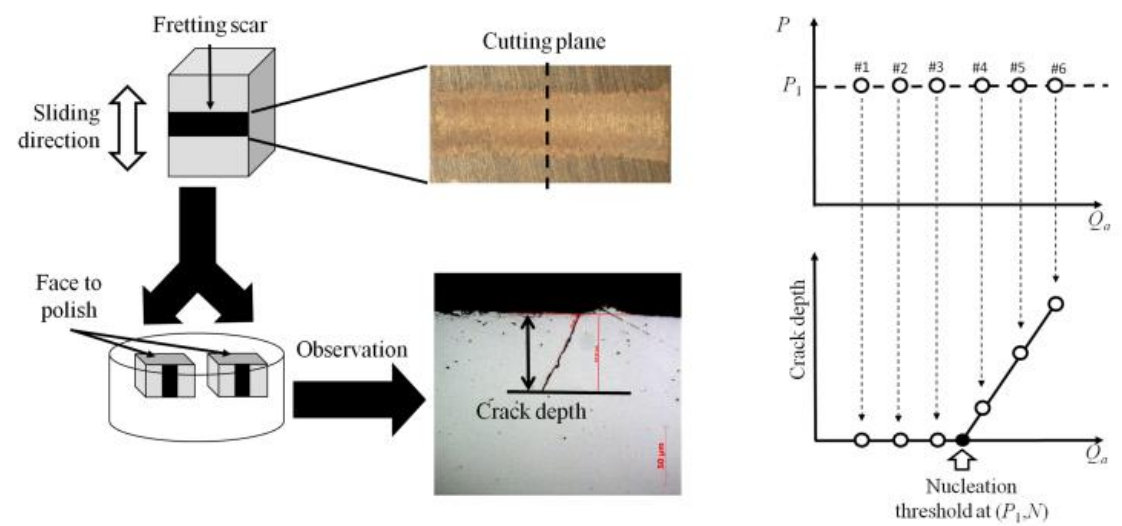

Fig. 3. Destructive crack nucleation identification technique

The maximum projected crack length $\left(b_{p, \max }\right)$ is plotted as a function of the applied tangential force amplitude $Q_{a}$. The crack nucleation threshold $Q_{d}(d$, for destructive) is then determined by extrapolating the tangential force amplitude at $b_{p, \max }=10 \mu \mathrm{m}$ (figure 4). All the tests were performed at $10^{6}$ cycles with a loading frequency of $10 \mathrm{~Hz}$. From 6 to 8 tests are usually required to estimate the crack threshold and more than 100 fretting experiments were performed to cover a wild range of loadings conditions. Therefore, this method is very time/material consuming.

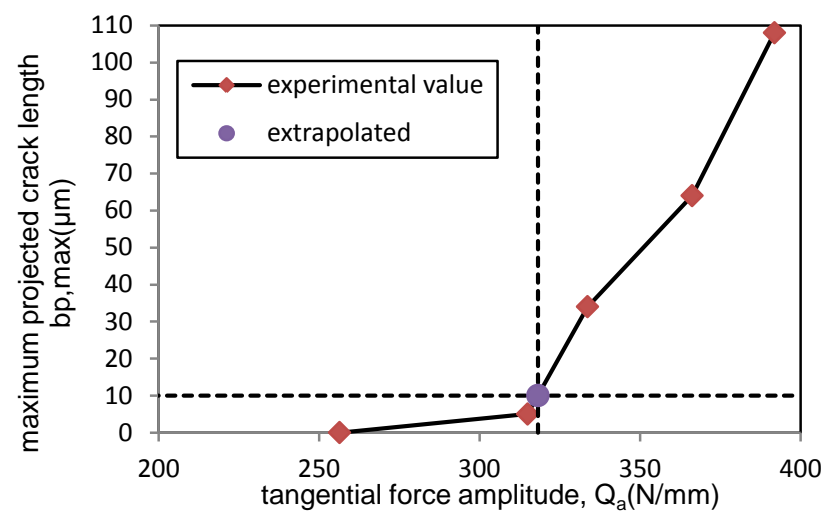

Fig. 4. Crack nucleation threshold identification for a given fretting condition 


\section{Coupled Kinematic-thermal identification technique: Non-destructive method}

The experimental set-up chosen for the coupled measurement was the two-face measurement set-up (figure 6). Employed for the first time in the works of $[12,13,14]$, the main advantage of this set-up is the flexibility to choose the surface coating of each technique separately. Some of the basic problems in this type of measurement are, the temporal matching of the two camera's and the spatial matching of CCD and IR images.

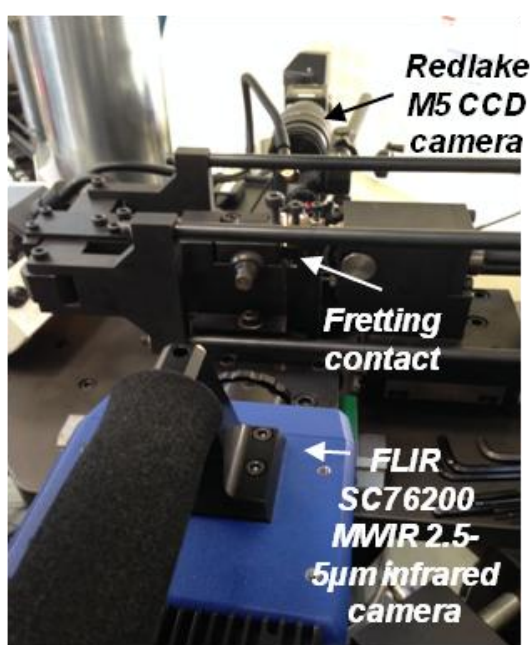

Fig. 6. The two-face experimental set-up

This section will be unfolded into four parts : Thermal measurement, synchronization (temporal matching), spatial matching, and non-destructive crack nucleation threshold method.

\subsection{Thermal measurement}

The camera used in this study is a FLIR SC7600 MWIR 2.5-5 $\mu$ m. The focal length of the optical lens is $25 \mathrm{~mm}$. This camera is equipped with an InSb $640 \times 512$ element detector. The maximal frame rate, $f_{a}$, is $380 \mathrm{~Hz}$ and the noise-equivalent temperature (NET) is lower than $25 \mathrm{mK}$. In this study, the size of a pixel is equal to $0.16 \times 0.16 \mathrm{~mm}^{2}$. The employed infrared camera should be first of all calibrated, this process is considered as the key parameter in the termographic measurement. Several calibration techniques can be found in literature, such as the non-uniformity correction [15], and the radiometric artefact treatment [16]. A pixel to pixel calibration process was used in this work as in [17]. A calibration law by detector pixel is calculated, connecting the DL (Digital Level) to the temperature through a recorded constant and uniform temperature field generated by a black body (Cl systems SR800R) (figure 7). The response of each pixel is approximated by a polynomial as below :

$$
T(i, j)=\sum_{p=0}^{p=P} a_{p}(i, j) D L(i, j)^{p}
$$

where $p$ is the polynomial degree, $a_{p}(i, j), D L(i, j)$ and $T(i, j)$ are respectively the calibration law coefficients, the digital level and the temperature of a pixel coordinate $(i, j)$.

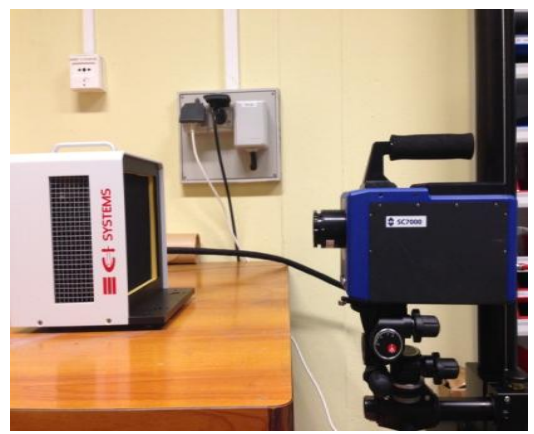

Fig. 7. IR camera facing the black body

Specimens are painted with a black matte paint to increase their emissivity. And the lens axis of the camera is kept fixed and held perpendicular to the lateral surface of the specimens (figure 8 (a)). Given the high thermal conductivity of the steel alloys, we can assume that the observed temperature field is very close to the temperature of the contact. For this study a macroscopic scale was chosen. At this scale, specimen deformations 
can be neglected. The temperature is then averaged over a Zone Of Interest (ZOI - figure 8 (b)). The size of this region is characterized by the Hertzian contact size $a$.

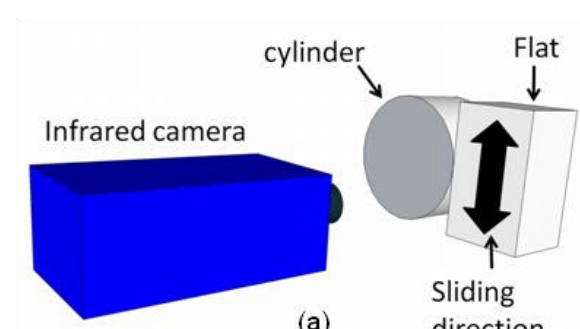

(a)

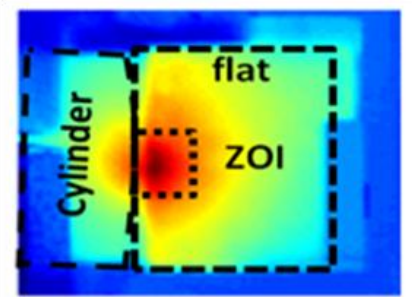

(b)

Fig. 8. (a) Basic sketch of the camera position (b) temperature field

Figure 9 shows the thermal response of the material during a simple Fretting test (same tendency for the fretting-fatigue tests) with constant loading parameters $\left(\mathrm{R}=80 \mathrm{~mm}, p_{\max }=800 \mathrm{MPa}, q_{\max }=520 \mathrm{MPa}\right.$, loading frequency $f_{L}=1 \mathrm{~Hz}$ and a camera frame rate $f_{a}=100 \mathrm{~Hz}$ ), averaged over the ZOI.

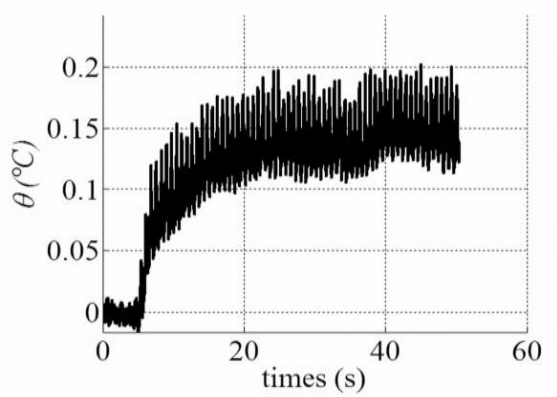

Fig. 9. Temperature variation evolution

We can observe a generalized warming of about $0.15^{\circ} \mathrm{C}$, and a temperature stabilization after a several cycles, superimposed by oscillatory variation of temperature with maximal amplitude of about $0.02^{\circ} \mathrm{C}$.

\subsection{Camera synchronization}

It is important to understand that each camera has its own physical time delay (the interval from receiving the signal to taking the image). And the accuracy of the coupled measurement cannot be guaranteed unless this time delay is known and taken into account. The time delay of the IR camera used in this study was indicated by the manufacturer $(2.8 \mu \mathrm{s})$. As for the CCD camera it was unknown and needed to be tested. Therefore, a free-fall experiment was done in order to measure this delay (Figure 10).

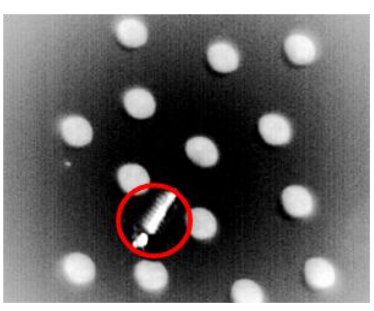

(a)

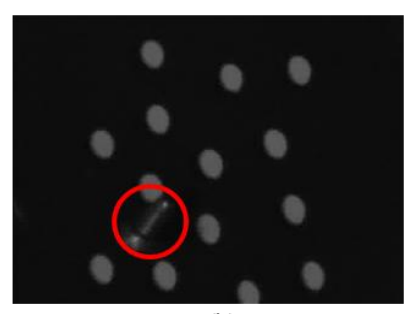

(b)

Fig. 10. Free-fall experiment: (a) The object in IR image, (b) visible image [18]

With the knowledge of the fall distance and the velocity of the object, abd by calculating its position difference in the two images, the real time delay of the CCD camera was estimated for 800 ms (a magnitude of $0.1 \mathrm{~ms}$ of error is considered acceptable in this study).

Now that the physical time delay of the cameras in known, a micro-controller (Arduino MEGA ADK) was employed to realize the synchronization. The working principle of the micro-controller is to trigger the two cameras, by sending two periodic pulse signals taking into account the time delay as shown in figure 11. 


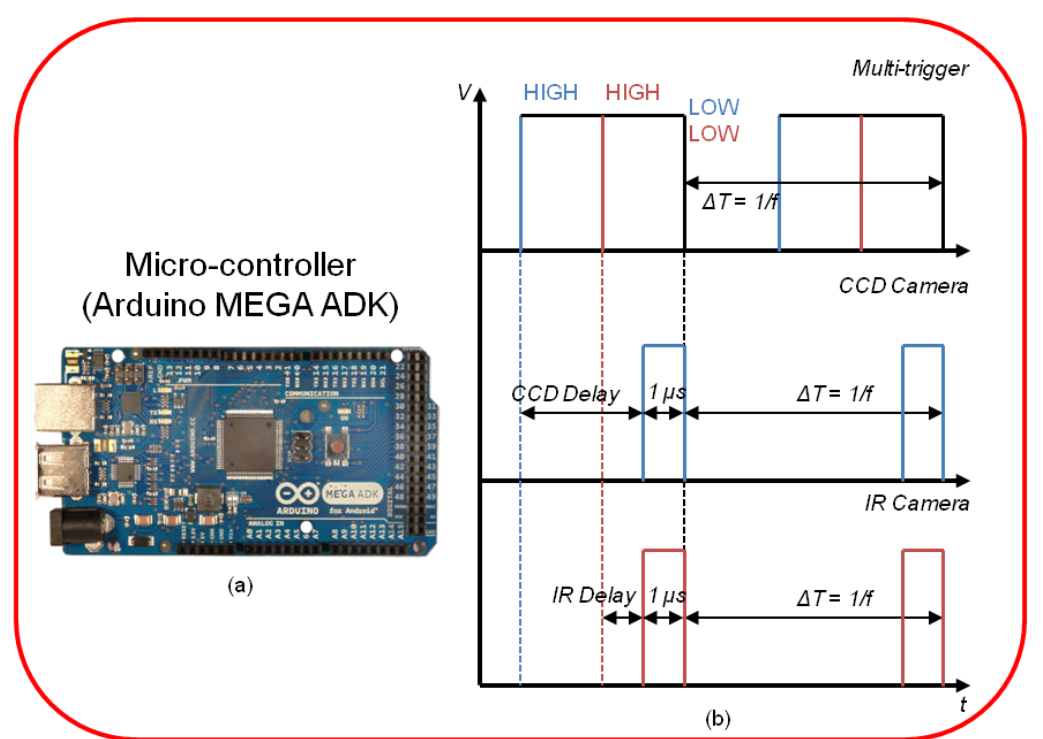

Fig. 11. (a) The Arduino MEGA ADK micro-controller, (b) The controlling flow [18]

\subsection{Spatial matching}

The conventional method for achieving a spatial matching on images acquired by two different cameras, is to make some physical marks on the object surface. the marks can then be recognized, by knowing the crossing function $\tau$ of points coordinates of an image to another. $\tau$ can be defined as follows (two translations and one rotation) :

$$
(\tau)\left\{\begin{array}{l}
x_{I R}=a_{x} x_{C C D}+b_{x} y_{C C D}+c_{x} \\
y_{I R}=a_{y} x_{C C D}+b_{y} y_{C C D}+c_{y}
\end{array}\right.
$$

In this study, a reference target (figure 12(a)) placed at exactly the same position where the specimen should be, was used in order to determine $\tau$.

(b)

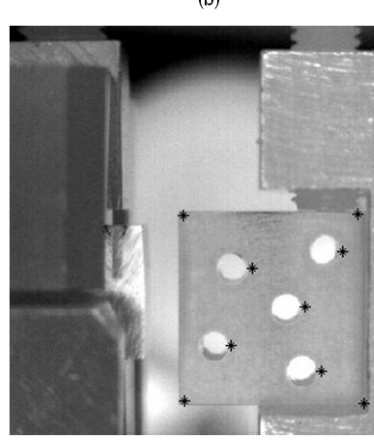

(c)

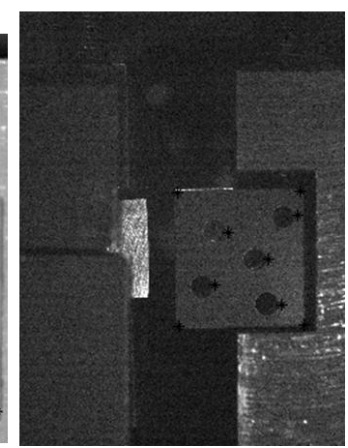

Fig. 12. (a) Reference target (b) IR image of the target (c) CCD image of the target

The images of the target are captured respectively by IR and CCD cameras. On each of these images, we identify at least three points geometrically identical (figure 12 (b) \& (c)). The point coordinates are then used to solve (2) with the least square method.

\subsection{Non-destructive crack nucleation threshold identification technique}

\subsubsection{Experiments parameters}

Tests in blocks of cycles with constant maximal Hertzian pressure, $p_{\max }$, and variable relative displacement amplitude, $\delta_{a}$, are made. the number of cycles per block should be sufficient to achieve temperature stabilization (at least 2000 cycles). For each block, when stabilized mechanical and thermal conditions were reached, $\delta_{a}$ was increased and then maintained constant until a new stable situation is attain (figure 13(a)). At each stabilized step, a coupled knimetic-thermal measurement is done (figure 13(b)). At least 200 images is taken for each step, with a loading frequency of $10 \mathrm{~Hz}$, and a sampling frequency of $19 \mathrm{~Hz}$. 


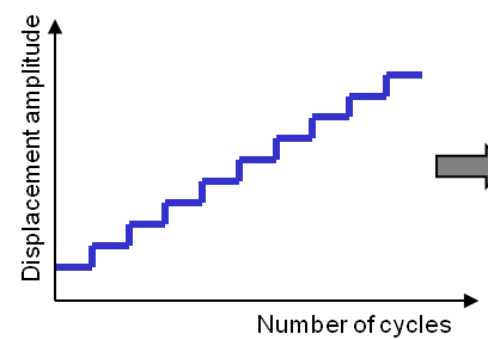

(a)

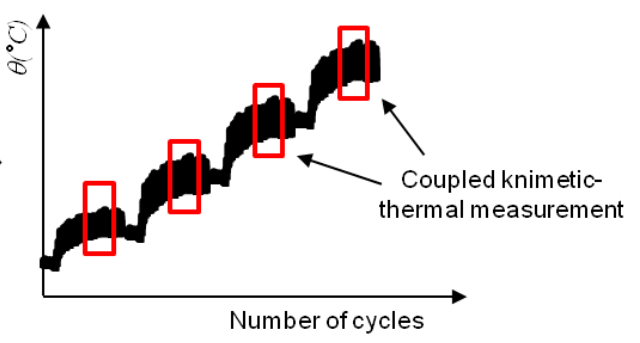

(b)

Fig. 13. (a) Principle of a variable displacement test, (b) coupled measurement

\subsubsection{Images processing}

Images processing was done as follows (figure 14): The ZOI is selected on the IR image, using the spatial matching function explaned above, the same zone is found on the CCD image. With the Digital Image Correlation (DIC) technique, displacements of the ZOI are calculated. In this work a macroscopic scale was chosen, at this scale specimens deformations can be neglected. Therfor, displacements obtained with the DIC are considered as the rigid body movement imposed by the experimental device and can be eliminated for a best estimation of the ZOI evolution.
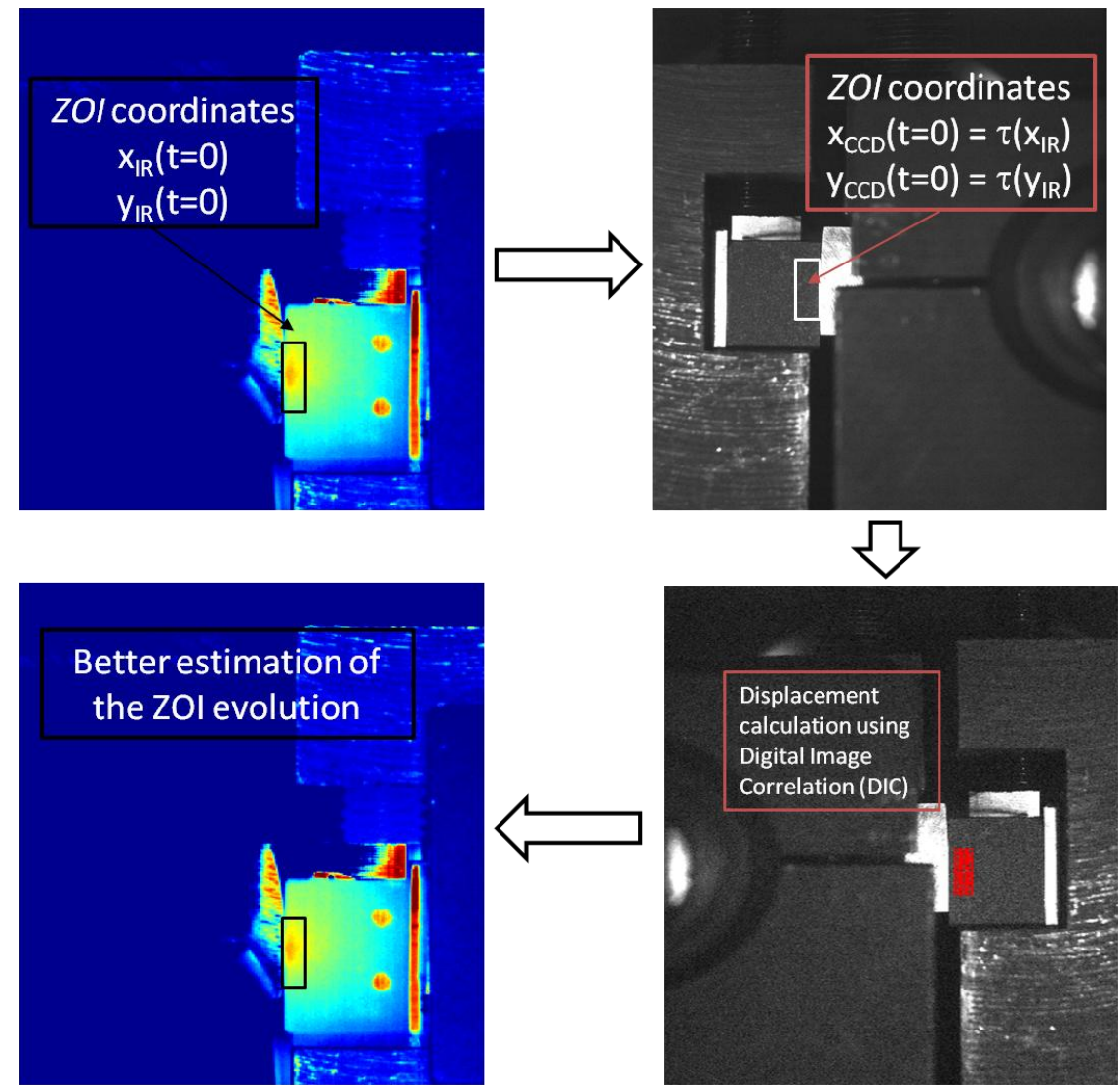

Fig. 14. Images processing

\subsubsection{Crack nucleation threshold identification using the thermal method}

In a previous study [19] we have showed that the temperature evolution averaged over a zone of interest (ZOI) can be decomposed into an overall thermal drift, and two periodic signals at $f_{L}$ and $2 f_{L}$, where $f_{L}$ is the loading frequency. Results showed also that the thermal drift and the amplitudes of each periodic function reach stabilized values $\left(\theta_{d}^{\text {sta }}, A_{f}^{\text {sta }}\right.$ and $\left.A_{2 f}^{\text {sta }}\right)$ after a few cycles (figure 15). 


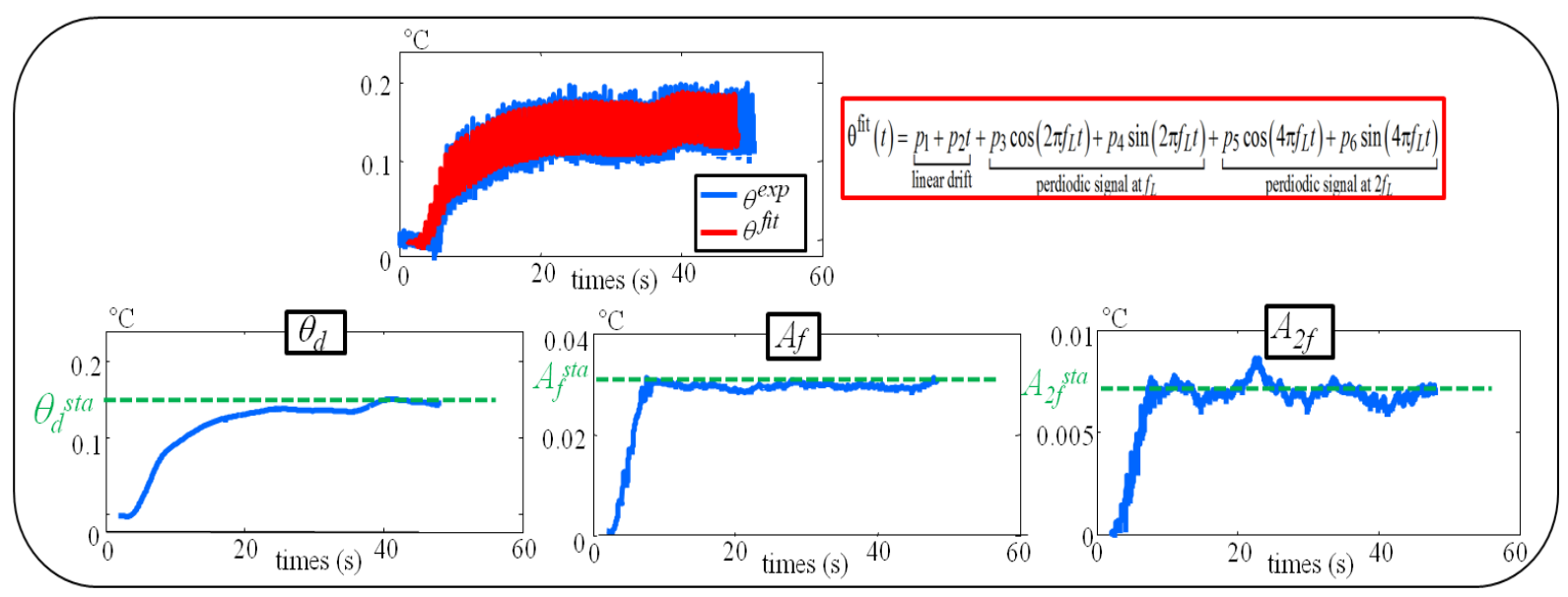

Fig. 15. Evolution of: $\theta^{\text {exp }}, \theta^{f i t}, \theta_{d}, A_{f}$ and $A_{2 f}$

Plotted as function of the maximum shear stress values $q_{\max }$, The rapid change in slope of these stabilized values, can be empiricaly connected with a critical maximal contact shear stress $\mathrm{q}_{\mathrm{TH}}$, ginving birth to a fretting crack. It is important to note that each stabilized function gives a different value of $q_{T H}$ (figure 16). Those values are then averaged and results will be presented in the next section. To have better understanding of this method please refer to [19].

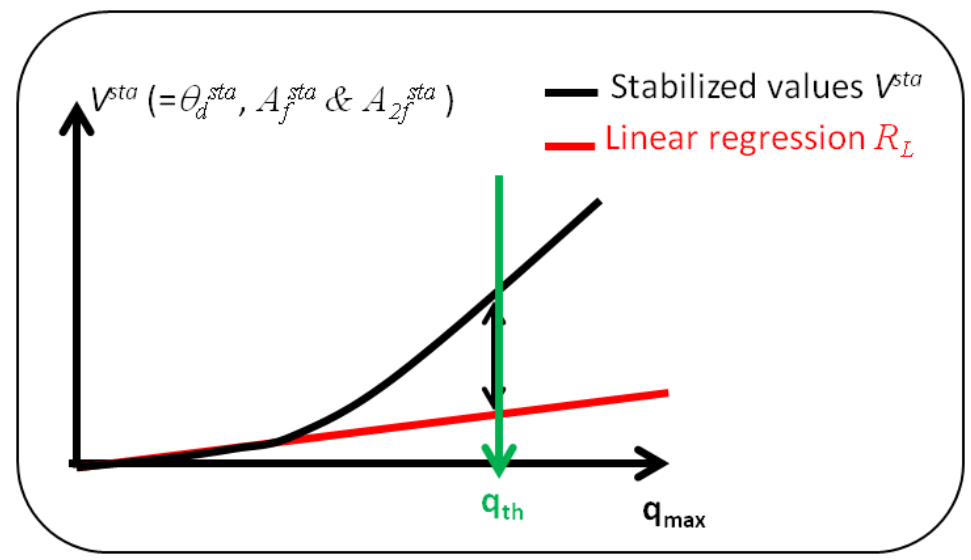

Fig. 16. Principle of the crack initiation threshold identification technique by mean of the thermal method

\section{Results and discussion}

One common approach to quantify fretting cracking response consists on reporting the crack nucleation boundaries in a $Q_{a}-P$ chart $\left(Q_{a}\right.$ for tangential force amplitude, $\mathrm{P}$ for normal loading), or a $Q_{a}-\sigma_{f a}$ chart ( $\sigma_{f a}$ for fatigue stress) for the fretting-fatigue mapping [20]. Such approach is appropriate to evaluate the effect of the normal force and the interactions between the fretting and the fatigue loadings. Fouvry et al. in [7] has developed a new approach to quantify the stress gradient effect. This approach consists on reporting the crack nucleation boundaries in a $\sigma_{f r}-P_{\max }\left(\sigma_{\mathrm{fr}}\right.$ for the contact stressing, $\mathrm{P}_{\max }$ for the maximal Hertzian pressure) and $\sigma_{f r}-\sigma_{f a}$ charts. figure 17 shows that keeping the maximum contact pressure constant, an increase in contact size due to larger cylinder radius will reduce the $\sigma_{f r}$ cracking stress. To interpret this evolution, we need to consider Neuber's theory [21] which shows that the crack nucleation process generated in a severe stress gradient configuration is controlled by the maximum stress state but also by the volume over which this maximum stressing condition is operating. The smaller the volume (i.e., the higher the stress gradient), the higher the cracking threshold values. figure 17 shows also a close correlation between results obtained from the thermal method and those obtained from the destructive one. 


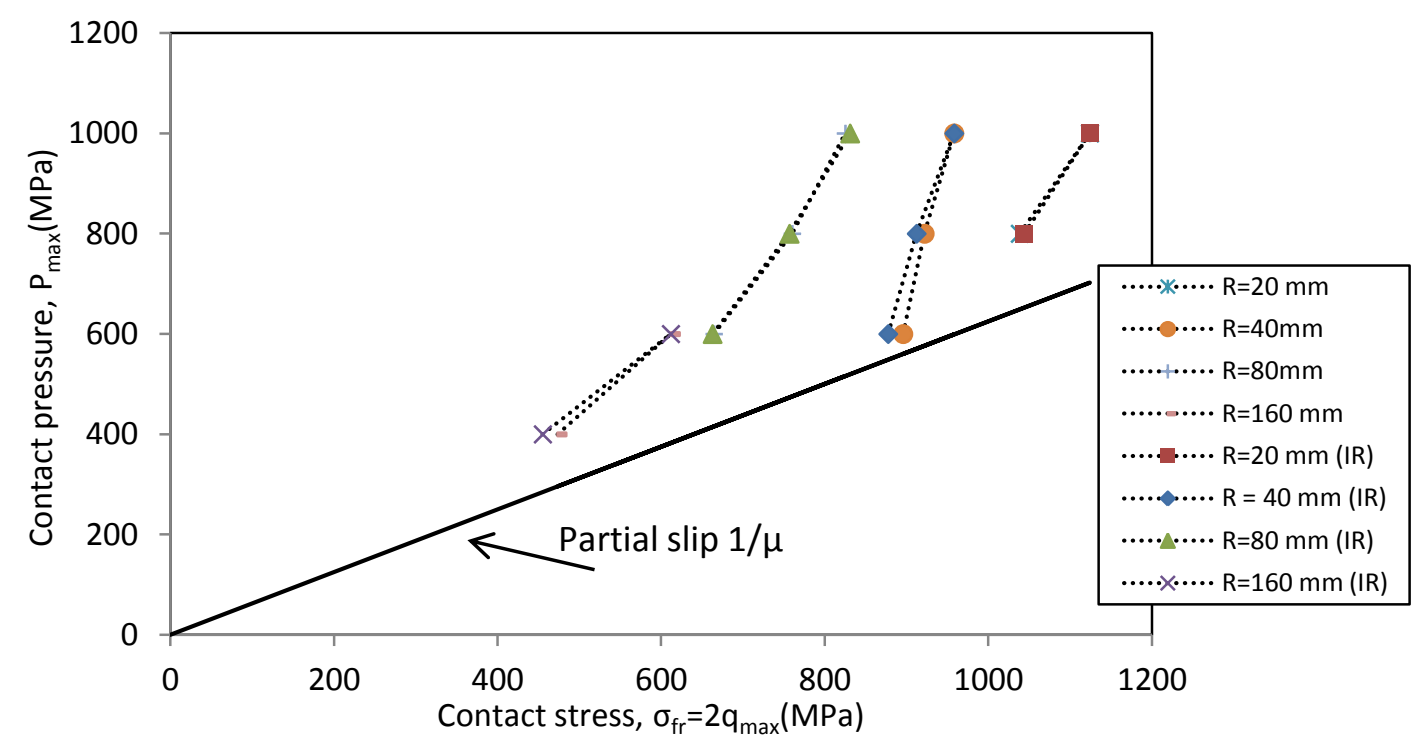

Fig. 17. $\sigma_{f r}-P_{\max }$ representation of the crack nucleation results by mean of the destructive and non-destructive methods ( $\mathrm{TH}$ for the non-destructive resutls)

A good correlation between both methods was also found in the case of the fretting-fatigue (figure 18) and the notched fatigue loadings (table 3 ).
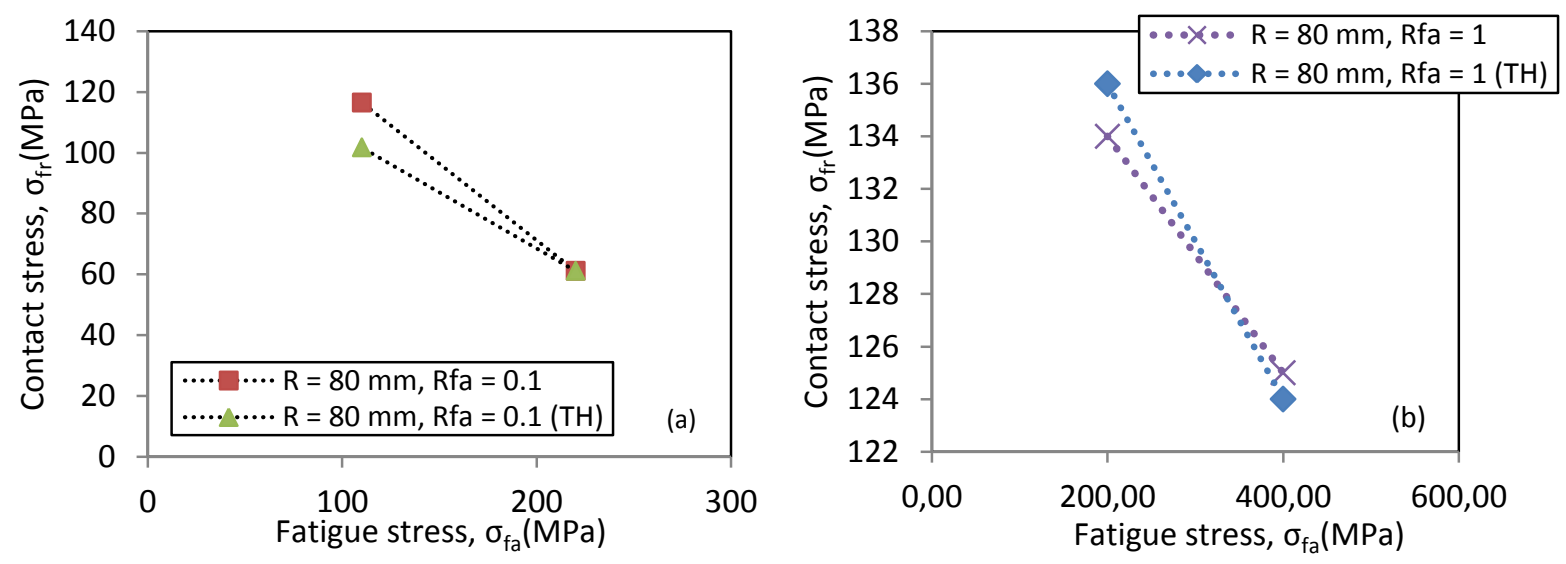

Fig. 18. $\sigma_{f r}-\sigma_{f a}$ representation of the crack nucleation results by mean of the destructive and non-destructive methods ( $\mathrm{TH}$ for the non-destructive results) : (a) $\mathrm{R}_{\mathrm{fa}}=0.1$, (b) $\mathrm{R}_{\mathrm{fa}}=1$.

\begin{tabular}{|c|c|c|}
\hline$r(m m)$ & $\sigma_{d}(\mathrm{MPA})$ & $\sigma_{\mathrm{TH}}(\mathrm{MPA})$ \\
\hline 2 & 212 & 206 \\
\hline
\end{tabular}

Table 3. Notched fatigue results

Figure 19 shows the relative error between both method for a fretting loading with cylinder radius of 80 $\mathrm{mm}$. For all other loadings conditions the error stays less than $5 \%$ and it is defined as follows :

$$
\text { error }=\left|\frac{q_{d}-q_{T H}}{q_{d}}\right|
$$




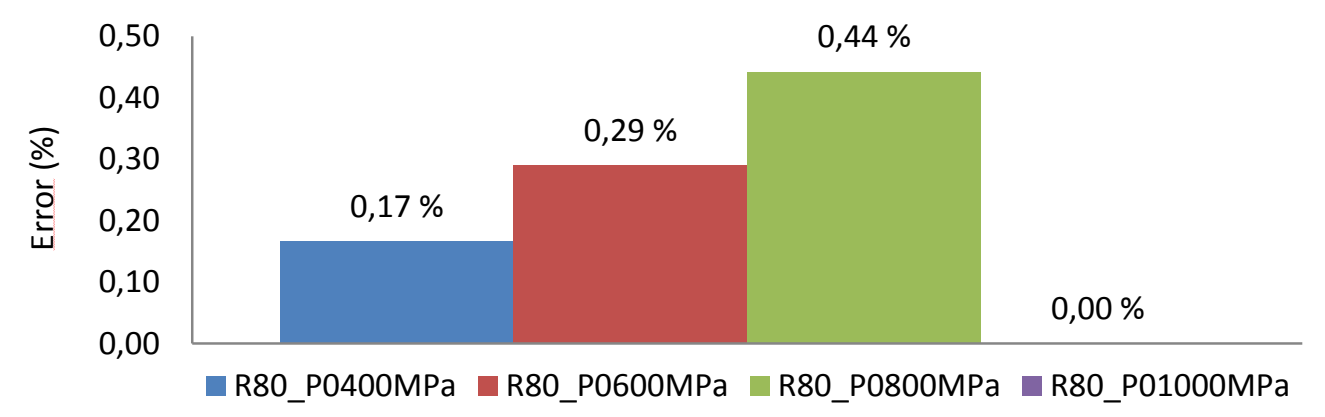

Fig. 19. Relative error between destructive and thermal methods for a cylinder radius of $80 \mathrm{~mm}$

\section{Conclusion}

In this study, an innovative experimental technique based on coupled kinematic and thermal measurements using an infrared and a CCD cameras was presented. One of the main advantages of this faceto-face set-up was the flexibility to choose the surface coating separately (black coating for the thermal measurements and a pattern for the DIC measurements). Problems related to temporal and spatial matching of images were solved using a multi-controller and a reference target.

This technique was then employed to determine the crack initiation threshold in different loading configurations, such as the fretting, the fretting-fatigue and notched fatigue loadings. Results have shown that the cracking phenomena generated in a very severe stress gradients situations are more important when cylinder radiuses are small (size effect).

A comparison between the classical destructive cracking identification technique and a non-destructive one was made and differences between both methods were less than $5 \%$. The new method presented in this work proved to be pertinent for different types of loadingslstress gradients situations.

\section{REFERENCES}

[1] Proudhon H., Fouvry S., and Yantio G., Determination and prediction of the fretting crack initiation: introduction of the $(P, Q, N)$ representation and definition of a variable process volume. International Journal of Fatigue 2006;28(7):707-713.

[2] Luong M. P., Fatigue limit evaluation of metals using an infrared thermographic technique. Mechanics of Materials 1998; 28(1-4):155-163.

[3] Moore HF, Kommers JB., Fatigue of metals under repeated stress. Chem Metall Eng 1921;25:1141-4.

[4] Krapez JC, Pacou D., Thermography detection of early thermal effects during fatigue tests of steel and aluminium samples. 29th Annuel review of progress in quantitative nondestructive evaluation. Brunswick, MN, USA: AIP; 2001; 1545-1552.

[5] La Rosa G, Risitano A., Thermographic methodology for rapid determination of the fatigue limit of materials and mechanical components. International Journal of Fatigue 2000;22;65-57.

[6] Doudard C., Poncelet M., Calloch S., Boue C., Hild F., and Galtier A., Determination of an HCF criterion by thermal measurements under biaxial cyclic loading. International Journal of Fatigue 2007;29(4):748757.

[7] Fouvry S., Gallien H., Berthel B., From uni- to multi-axial fretting-fatigue crack nucleation: Development of a stress-gradient-dependent critical distance approach. International Journal of Fatigue 2014;62:194209.

[8] Fouvry S., Kapsa Ph., Sidoroff F., Vincent L., Identification of the characteristic length scale for fatigue cracking in fretting contacts. J Phys IV France 1998;8:159-66.

[9] Meriaux J., Fouvry S., Kubiak KJ., Deyber S., Characterization of crack nucleation in TA6V under fretting-fatigue loading using the potential drop technique. Int J Fatigue 2010;31(10):1658-68.

[10] Fellows LJ., Nowel D., Hill DA., On the initiation of fretting fatigue cracks. Wear 1997;205:120-9.

[11] Lee H., Mall S., Investigation into effects and interaction of various fretting fatigue variables under slipcontrolled mode. Tribol Int 2006;39:1213-9.

[12] Chrysochoos A., Berthel B., Latourte F., Galtier A., Pagano S., Wattrisse B., Local energy analysis of high-cycle fatigue using digital image correlation and infrared thermography. The Journal of Strain Analysis for Engineering Design 2008a;43(6):411-422.

[13] Chrysochoos A., Berthel B., Latourte F., Galtier A., Pagano S., Wattrisse B., Local energy approach to steel fatigue. Strain 2008b;44(4):327-334.

[14] Chrysochoos A., Huon V., Jourdan F., Muracciole J., Peyroux R., Wattrisse B., Use of full-field Digital Image Correlation and infrared thermofrapgy measurements for the thermomechanical analysis of material behaviour. Strain 2010;45(1):117-130.

[15] Poncelet M., Witz J-F., Pron H., Wattrisse B., A study of IRFPA camera measurement errors: radiometric artefacts. Quantitative InfraRed Thermography Journal 2011;8(1):3-20. 
[16] Schulz M., Caldwell L., Nonuniformity correction and correctability of infrared focal plane arrays. Infrared Physics and Technology 1995;36(4):763-77.

[17] Honorat V., Moreau S., Muracciole J. M., Wattrisse B., and Chrysochoos A., Calorimetric analysis of polymer behaviour using a pixel calibration of an IRFPA camera. J. Quant. InfraRed Thermogr. 2005; 2(2):153-171.

[18] Wang X.G., Witz J-F., El Bartali A., Dufrénoy P., Charkaluk E., Investigation of grain-scale surface deformation of a pure aliminium polycrystal through kinematic-thermal full-field coupling measurement. 13th International Conference on Fracture 2013.

[19] Berthel B., Moustafa A-R., Charkaluk E., Fouvry S., Crack nucleation threshold under fretting loading by a thermal method. Tribology International (2013), http://dx.doi.org/10.1016/j.triboint.2013.10.008.

[20] Proudhon H., Fouvry S., Buffière J-Y., A fretting crack initiation prediction taking into account the surface roughness and the crack nucleation process volume. Int J Fatigue 2005;27(5):569-79.

[21] Neuber H. Theory of notch stresses. Berlin: Springer; 1958. 